\title{
ASOCIACIÓN ENTRE PARÁMETROS ANTROPOMÉTRICOS Y CARIES DENTAL EN NIÑOS ENTRE 5 A 6 AÑOS DE LIMA
}

\section{ASOCIATION BETWEEN ANTHROPOMETRIC PARAMETERS AND DENTAL CARIES AMONG CHILDREN FROM 5 TO 6 YEARS OF LIMA}

\author{
Ana Cullash-Luza'a ${ }^{1 a}$ Eraldo Pesaressi-Torres ${ }^{1 b}$, Rafael Morales-Vadilloc, Silvia Luza-Monterod
}

\section{RESUMEN}

Objetivos: El propósito del presente estudio fue determinar la asociación entre los parámetros antropométricos y la prevalencia de caries dental y sus consecuencias clínicas en niños de la Institución Educativa Inicial 064 "Señor de los Milagros" de Santa Anita, Lima. Materiales y Métodos: El estudio fue analítico y transversal en 100 niños de 5 y 6 años de edad. El diagnóstico de caries dental y sus consecuencias clínicas se realizó mediante criterios OMS y el índice de consecuencias clínicas de caries dental no tratada (pufa). Los parámetros antropométricos de peso para la edad, talla para la edad e índice de masa corporal para la edad fueron determinados con el software AnthroPlus (OMS). Se analizó la asociación entre estas variables con la prueba Chi-cuadrado. Resultados: Se encontró una alta prevalencia de caries dental (73\%) y sus consecuencias clínicas (33\%). Se detectó un incremento de la prevalencia de pufa en los niños de 6 años de edad ( $p=0,013)$. La mayoría de los niños presentaron un peso adecuado (88\%), mientras que el $7 \%$ y $4 \%$ presentaron sobrepeso u obesidad, respectivamente. Los promedios de los indicadores antropométricos fueron ligeramente menores en el grupo de alta prevalencia de caries dental; sin embargo, no se encontraron diferencias significativas. Conclusiones: La muestra evaluada presenta una alta prevalencia de caries, sobretodo en el grupo clasificado como normal. No se encontró asociación entre los parámetros antropométricos y la prevalencia de caries dental y sus consecuencias clínicas en los niños de la muestra.

Palabras clave: antropometría; preescolar; caries dental; salud pública. (Fuente: DeCS)

\section{ABSTRACT}

Objective: The aim of this study was to determine the association between anthropometric parameters and the prevalence of dental caries and its clinical consequences among children from the Señor de los Milagros preschool in Santa Anita, Lima. Materials and Methods: In this analytical cross-sectional study, 100 children from 5 to 6 years of age were examined. Dental caries was registered with the WHO's criteria, clinical consequences of untreated dental caries were assessed with the pufa index and anthropometric indexes (weight for age, height for age and BMI for age) were measured using WHO's AnthroPlus software. The association between dmf-t index and BMl was then analyzed; the data was processed by Chi-square test. Results: A high prevalence of dental caries (73\%) and its clinical consequences (33\%) was found in this preschool population. An increment in the pufa index was detected in children aged $6(p=0,013)$. Most of the children were in the healthy weight category (88\%), while the $7 \%$ and $4 \%$ were overweight and obese, respectively. Anthropometric measures were slightly lower in the high caries prevalence group, but no significant differences were found. Conclusions: These children have a very high prevalence of dental caries, especially among a group classified as healthy. There was no association between dental caries and its clinical consequences and anthropometric parameters in this preschool population.

Key words: anthropometry; child, preschool; dental caries; public health. (Source: MeSH NLM)

${ }^{1}$ Facultad de Odontología, Universidad San Martín de Porres, Lima, Perú.

${ }^{2}$ Universidad Alas Peruanas. Lima, Perú.

a Cirujano Dentista USMP,

${ }^{b}$ Docente del curso de Odontopediatría

c Director del Instituto de Investigación

d Docente del curso de Clínica Integral de Adulto

Correspondencia:

Ana Claudia Cullash Luza

Correo electrónico: claudiacluza@gmail.com

Citar como: Cullash A, Pesaressi E, Morales R, Luza S. Asociación entre parámetros antropométricos y caries dental en niños entre 5 a 6 años de Lima. KIRU. 2017;14(2): 135 - 143. doi:10.24265/kiru.2017.v2n2.5 


\section{INTRODUCCIÓN}

La caries dental es una enfermedad crónica y multifactorial, común en la infancia y que no distingue edad, género ni estrato social o cultural. Representa un problema relevante dentro del ámbito de la salud pública debido a su alta prevalencia a nivel mundial y las consecuencias en la calidad de vida de quienes la padecen ${ }^{(1,2)}$. En la población escolar peruana, los estudios reportan un promedio de seis piezas cariadas por persona ${ }^{(3)}$.

La caries dental y la desnutrición/obesidad en la infancia son condiciones con etiología multifactorial y componentes ambientales y genéticos predisponentes. Tanto la desnutrición como la obesidad resultan de un desequilibrio entre la ingesta proteico-calórica y los requerimientos energéticos del cuerpo, con implicaciones adversas significativas para la salud a corto y largo plazo(4-7)

Las lesiones de caries dental no tratadas tienen un impacto negativo en la adecuada nutrición y el crecimiento de los niños, ya que comúnmente se asocian a dolor e incomodidad ${ }^{(8-11)}$. La cronicidad de la enfermedad podría tener el mismo efecto sobre el crecimiento normal del niño que cualquier otra enfermedad crónica o infección, representando un riesgo potencial para la salud y bienestar general infantil.

Estudios realizados a nivel mundial intentaron establecer una asociación entre niveles altos de caries dental y una reducción de los valores antropométricos en la infancia, con resultados no concluyentes ${ }^{(12,13)}$. La mayoría de niños pequeños con lesiones severas de caries dental presentaron un crecimiento deficiente y una ganancia de peso reducida ${ }^{(14-19)}$. Una teoría tras esta situación propone que la interrupción del sueño profundo, asociada al dolor o infección por caries dental, puede inducir la inhibición de la liberación de hormona de crecimiento $(\mathrm{GH})$. Por otro lado, las lesiones no tratadas de caries dental producen dolor por su rápido avance y destrucción de tejidos, limitando al niño en su capacidad de alimentarse con normalidad y reduciendo la ingesta calórica y de nutrientes necesarios para un adecuado desarrollo ${ }^{(20,21)}$.

Estudios adicionales en poblaciones escolares reportaron que no existe asociación entre estas variables ${ }^{(22-25)}$, probablemente debido a limitaciones metodológicas $^{(13)}$, mientras que investigaciones longitudinales han descrito sus resultados con respecto a los beneficios del tratamiento de la caries dental y la consecuente mejora en el peso, talla e índice de masa corporal de los niños atendidos ${ }^{(26-28)}$.
En contraste a estos estudios, diferentes autores reportaron que niños obesos presentaron alta prevalencia de caries dental ${ }^{(29,30)}$, indicando que esta situación podría deberse a los cambios dietéticos caracterizados por el consumo excesivo deproductos con alta carga energética que a la vez son factores de riesgo de caries dental y obesidad, no solo en países desarrollados, sino también en las poblaciones menos favorecidas $^{(31,4,5)}$.

Actualmente ha surgido un gran interés en describir el impacto de las enfermedades orales en la salud general del individuo ${ }^{(12,15,32)}$. La relación entre caries dental y crecimiento físico es compleja ya que involucra una serie de factores genéticos, socioeconómicos y culturales de las poblaciones $^{(1,2,4,5)}$. En nuestro país, el impacto de la caries dental no tratada sobre el crecimiento físico y la salud infantil es un tema poco estudiado y la evidencia es limitada y controversial ${ }^{(33,34)}$. Es necesario conocer el estado actual de esta problemática, considerando los grupos con mayor riesgo, para intervenir con estrategias de rehabilitación, pero sobre todo de prevención, que promuevan el bienestar de la población preescolar.

El objetivo del presente estudio fue determinar la asociación entre los parámetros antropométricos y la prevalencia de caries dental y sus consecuencias clínicas en niños de 5 y 6 años de la Institución Educativa Inicial 064 "Señor de los Milagros" en Santa Anita, Lima.

\section{MATERIALES Y MÉTODOS}

Considerando el objetivo de la investigación, se diseñó un estudio observacional, analítico y de corte transversal. Fueron examinados 100 niños de la Institución Educativa Inicial "Señor de los Milagros"Santa Anita, seleccionados por un procedimiento de muestreo no probabilístico por conveniencia. Cabe señalar que solo fueron considerados aquellos niños que colaboraron con el examen bucal y contaron con el consentimiento de sus padres. Los criterios de exclusión fueron: niños con condiciones perinatales y hábitos alimenticios que alteren su peso o talla al momento de realizar las mediciones o que faltaron por alguna razón.

La operadora recibió soporte teórico y práctico para luego establecer una calibración con 10 evaluaciones en pacientes, obteniéndose el valor del coeficiente Kappa para la consistencia inter-examinador de 0.906 . Se programó una actividad de promoción de salud en las instalaciones del colegio dirigida a los padres de familia, tutores de los niños y docentes, par a 
informarles del estudio y solicitarles la firma del consentimiento informado que autoriza la participación de sus hijos en el estudio, cubriendo así los aspectos éticos y legales de la investigación (Figura 1).

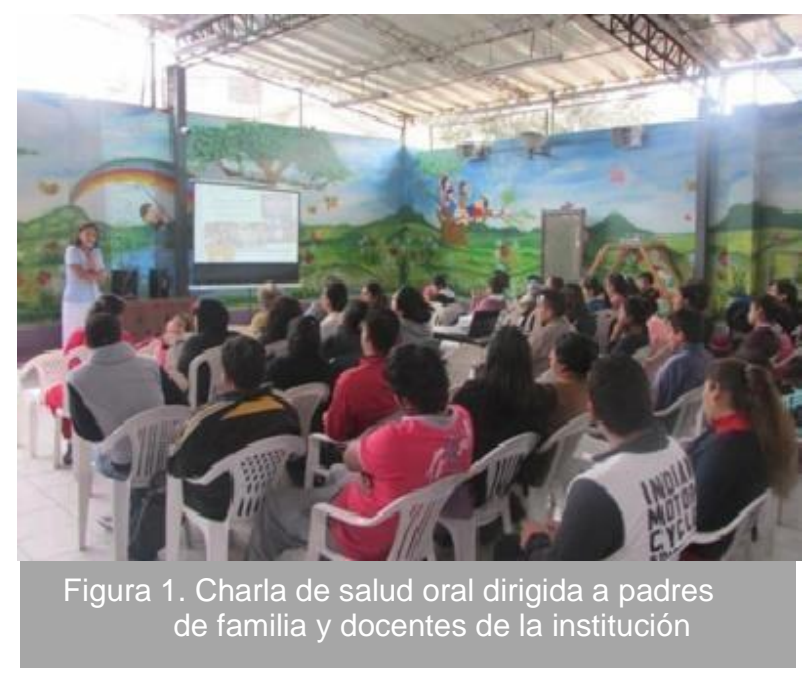

Los datos personales de cada niño, la evaluación bucodental, peso y talla fueron registrados. El examen bucodental se realizó con instrumental de diagnóstico clínico básico (espejos bucales y exploradores con punta roma), sillas, una mesa y luz artificial blanca. Se utilizaron guantes desechables, gorro, mascarilla y lentes protectores.

Luego del cepillado dental, se observó la presencia de caries como cambio de coloración (mancha gris, marrón u oscura) o cavitación, bajo los criterios establecidos por la OMS. Debido a la edad de los niños evaluados y la consecuente baja o nula prevalencia de caries en la dentición permanente, se hizo énfasis en la experiencia de caries en la dentición primaria. Asimismo, se utilizó el índice de consecuencias clínicas de caries dental no tratada (pufa). El índice pufa fue propuesto en 2010 por Monse B et al. ${ }^{(35)}$, con la intención de evaluar diferentes etapas avanzadas de la lesión cariosa y así complementar a los índices de caries dental existentes. Registra la presencia de dientes deciduos severamente cariados con afectación visible de la pulpa $(p)$, ulceración de la mucosa oral por fragmentos de raíz $(u)$, presencia de fístula $(f)$ y la presencia de abceso $(a)$.

La evaluación antropométrica se realizó según los procedimientos establecidos por la OMS. Los niños fueron evaluados descalzos y con un mínimo de ropa. Para pesar a los niños se usó una balanza digital (Beurer, Alemania) y dicha medición se realizó en el mismo momento del día y antes de ingerir el refrigerio. El peso se registró en kilogramos $(\mathrm{kg})$ hasta el $0,5 \mathrm{~kg}$ más próximo. Para medir la estatura, el niño se posicionó derecho, descalzo sobre el instrumento a utilizar, un tallímetro (ADE, Alemania). La talla se registró en centímetros $(\mathrm{cm})$, hasta el 0,5 más próximo. Con los datos de las mediciones se obtuvieron los puntajes Z para los indicadores de peso para la edad, talla para la edad e índice de masa corporal para la edad con la herramienta de calculadora antropométrica, proporcionada por el software Anthro Plus 1.0 .4 de la OMS. La interpretación de los puntajes $Z$ obtenidos se realizó con los criterios establecidos por las nuevas referencias internacionales de la OMS ${ }^{(36)}$.

Los datos se procesaron mediante un análisis estadístico con la prueba de Chi cuadrado para evaluar la asociación entre los parámetros antropométricos y la prevalencia de caries dental y sus consecuencias clínicas. Para el análisis entre los promedios de los indicadores antropométricos según la prevalencia y severidad de caries dental además se emplearon las pruebas de Kruskall-Wallis, ANOVA, $t$ Student y U de Mann-Whitney. El análisis de losdatos se realizó con el programa SPSS versión 23 en español (IBM-Estados Unidos).

El estudio contó con la aprobación del Comité de Ética de la Facultad de Odontología de la USMP.

\section{RESULTADOS}

Se evaluaron 100 niños de 5 y 6 años de edad, 51 del sexo femenino y 49 del sexo masculino. En cuanto a la edad, 54 tenían 5 años y 46, 6 años.

El $73 \%$ de los niños de la muestra presentó caries dental y el índice ceo-d promedio fue 4,18 (DS 4,118) con un valor mínimo y máximo de 0 y 18 , respectivamente. El $47 \%$ de los niños examinados presentó una baja prevalencia de caries dental, seguida de la prevalencia alta (29\%) y moderada (24\%). (Tabla 1).

Tabla 1. Niveles de severidad de prevalencia de caries dental en niños entre 5 a 6 años.

\begin{tabular}{ccc}
\hline ceo-d & Frecuencia & Porcentaje \\
\hline bajo/muy bajo (0-2) & 47 & $47 \%$ \\
moderado (3-6) & 24 & $24 \%$ \\
alto (7 o más) & 29 & $29 \%$ \\
\hline Total & 100 & $100 \%$
\end{tabular}


El $33 \%$ de niños evaluados presentó alguna consecuencia clínica de caries dental no tratada (índice pufa). Se observó un incremento en la prevalencia del índice pufa en los niños de 6 años
$(45,7 \%)$, en comparación con los niños de 5 años $(22,2 \%)$. Esta diferencia fue estadísticamente significativa ( $p=0,013)$ (Figura 2$)$.

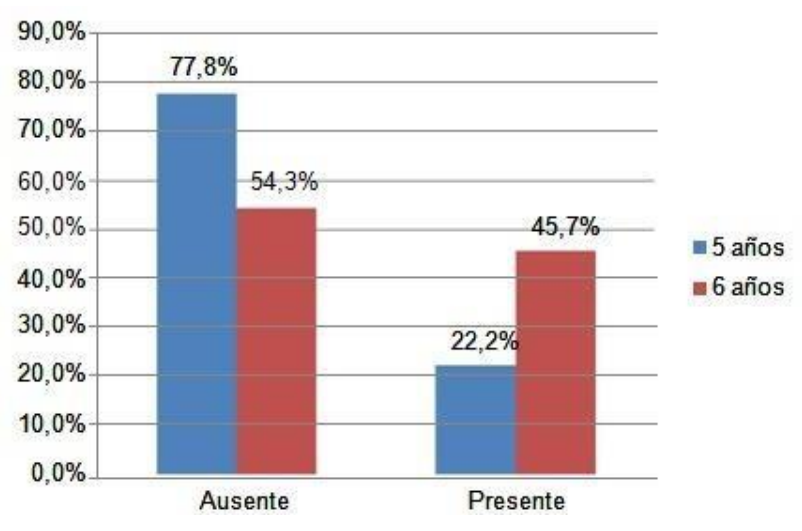

Figura 2. Prevalencia de consecuencias clínicas de caries dental no tratada según edad.

INDICE PUFA

La valoración nutricional según la OMS (indicador IMC/edad) reportó un bajo porcentaje de obesidad (4\%). En cuanto al indicador de peso para a la edad, observamos que del total de 100 niños evaluados, 88 presentaron un peso adecuado siendo esta prevalencia mayor en los niños de 5 años (53,34\%) que en los de 6 años ( $46.6 \%$ ). Se encontró que el $7 \%$ de los niños estaban en la categoría de alto peso, $4 \%$ tenian riesgo de sobre peso y sólo un caso de bajo peso. (Tabla 3).

Tabla 2. Indicador de índice de masa corporal para la edad de los niños

\begin{tabular}{|c|c|c|c|c|c|c|}
\hline \multicolumn{7}{|c|}{ Edad } \\
\hline \multirow[t]{2}{*}{ IMC/edad } & \multicolumn{2}{|c|}{5 años } & \multicolumn{2}{|c|}{6 años } & \multicolumn{2}{|c|}{ Total } \\
\hline & $\mathbf{n}$ & $\%$ & $\mathbf{n}$ & $\%$ & $\mathbf{n}$ & $\%$ \\
\hline Bajo peso & 1 & $100,0 \%$ & 0 & $0,0 \%$ & 1 & $100,0 \%$ \\
\hline Peso adecuado & 47 & $53,4 \%$ & 41 & $46,6 \%$ & 88 & $100,0 \%$ \\
\hline Alto peso & 5 & $71,4 \%$ & 2 & $28,6 \%$ & 7 & $100,0 \%$ \\
\hline Riesgo de sobre & 1 & $25,0 \%$ & 3 & & & $100,0 \%$ \\
\hline peso & & & & $75,0 \%$ & 4 & \\
\hline Total & 54 & $54,0 \%$ & 46 & $46,0 \%$ & 100 & $100,0 \%$ \\
\hline
\end{tabular}

En las categorías de P/E y T/E adecuadas para la edad, el 46,7\% de los niños presentó un índice ceo-d bajo. El $47,7 \%$ de niños en la categoría de IMC/E adecuado presentó un índice ceo-d bajo (Tabla 3). 
Tabla 3. Indicador de imc/edad según niveles de severidad de caries dental

\begin{tabular}{|c|c|c|c|c|c|c|c|c|}
\hline \multirow{3}{*}{ IMC/edad } & \multicolumn{8}{|c|}{ CEO-D } \\
\hline & \multicolumn{2}{|c|}{ bajo/muy bajo } & \multicolumn{2}{|c|}{ moderado } & \multicolumn{2}{|c|}{ alto } & \multicolumn{2}{|c|}{ Total } \\
\hline & $\mathrm{n}$ & $\%$ & $\mathrm{n}$ & $\%$ & $\mathrm{n}$ & $\%$ & $\mathbf{n}$ & $\%$ \\
\hline Bajo peso & 1 & $100,0 \%$ & 0 & $0,0 \%$ & 0 & $0,0 \%$ & 1 & $100,0 \%$ \\
\hline $\begin{array}{c}\text { Peso } \\
\text { adecuado }\end{array}$ & 42 & $47,7 \%$ & 19 & $21,6 \%$ & 29 & $30,7 \%$ & 88 & $100,0 \%$ \\
\hline Alto peso & 3 & $42,9 \%$ & 3 & $42,9 \%$ & 2 & $14,3 \%$ & 7 & $100,0 \%$ \\
\hline Muy alto & 1 & $25,0 \%$ & 2 & $50,0 \%$ & & & & $100,0 \%$ \\
\hline peso & & & & & 2 & $25,0 \%$ & 4 & \\
\hline Total & 47 & $47,0 \%$ & 24 & $24,0 \%$ & 33 & $29,0 \%$ & 100 & $100,0 \%$ \\
\hline
\end{tabular}

Con respecto a la prevalencia de consecuencias clínicas de caries dental no tratada, el $67 \%$ de niños en la categoría de IMC/E adecuado se encontró libre de estas complicaciones. (Tabla 4)

Tabla 4. Indicador de peso/edad según prevalencia de consecuencias clínicas de caries dental.

\begin{tabular}{|c|c|c|c|c|c|c|}
\hline \multirow{3}{*}{ PESO } & \multicolumn{5}{|c|}{ PUFA } & \\
\hline & \multicolumn{2}{|c|}{ Ausente } & \multicolumn{2}{|c|}{ Presente } & \multicolumn{2}{|c|}{ Total } \\
\hline & $\mathrm{n}$ & $\%$ & $\mathrm{n}$ & $\%$ & $\mathbf{n}$ & $\%$ \\
\hline Bajo peso & 1 & $100,0 \%$ & 0 & $0,0 \%$ & 1 & $100,0 \%$ \\
\hline $\begin{array}{c}\text { Peso } \\
\text { adecuado }\end{array}$ & 51 & $68,0 \%$ & 24 & $32,0 \%$ & 75 & $100,0 \%$ \\
\hline $\begin{array}{l}\text { Riesgo de } \\
\text { alto peso }\end{array}$ & 11 & $64.7 \%$ & 6 & $35,3 \%$ & 17 & $100,0 \%$ \\
\hline Sobre peso & 4 & $57.1 \%$ & 3 & $42,9 \%$ & 7 & $100,0 \%$ \\
\hline & 67 & $67,0 \%$ & 3 & $33,0 \%$ & 100 & $100,0 \%$ \\
\hline
\end{tabular}

Los promedios de las medidas antropométricas fueron ligeramente menores en el grupo de alta prevalencia de caries dental; sin embargo, no se encontraron diferencias significativas. (Tabla 5). 
Tabla 5. Promedios de indicadores antropométricos estudiados según niveles de prevalencia de caries dental

\begin{tabular}{lcccc}
\multicolumn{5}{c}{ CEO- M } \\
& BAJO & MODERADO & ALTO \\
Peso & Media (D.E.) & Media (D.E.) & Media (D.E.) & $p$-valor \\
Talla & $21,31(2,81)$ & $22,53(3,64)$ & $21,2(3,65)$ & $0,341 \mathrm{a}$ \\
IMC & $112,56(3,81)$ & $113,25(4,20)$ & $112,43(4,80)$ & $0,752 \mathrm{~b}$ \\
& $16,82(2,01)$ & $17,6(2,93)$ & $16,66(1,92)$ & $0,416 \mathrm{a}$
\end{tabular}

a: Prueba de Kruskall-Wallis

b: ANOVA de una vía

\section{DISCUSIÓN}

En la actualidad, la relación entre parámetros antropométricos y caries dental en la dentición decidua es compleja y controversial, con resultados de investigación no concluyentes con respecto su existencia y naturaleza ${ }^{(13)}$. Algunos estudios reportaron que niños con niveles más altos de caries dental presentaron un crecimiento insuficiente ${ }^{(14-19)}$, mientras que otros encontraron que un IMC alto se asocia a mayor prevalencia de caries dental infantil no tratada(29-31). Sin embargo, existen otros que no reportaron ninguna asociación entre estas variables $(23-25,37,38)$.

El presente estudio no encontró asociación estadísticamente significativa entre los parámetros antropométricos y la prevalencia/severidad de caries dental de los niños de 5 y 6 años de la Institución Educativa Inicial 064 "Señor de los Milagros", de acuerdo con los antecedentes revisados ${ }^{(23,28)}$. Cabe resaltar que la población estudiada presentó en su mayoría niños con un estado nutricional normal (IMC/E adecuado), coincidiendo con lo reportado por estudios previos en poblaciones similares ${ }^{(22,33,34)}$.

Al observar la prevalencia de las otras categorías de estado nutricional, el sobrepeso/obesidad se encontró en un porcentaje mayor (11\%) que la de bajo peso (1\%); situación que podría explicarse por los cambios dietéticos en países en vías de desarrollo, como el consumo excesivo de productos industrializados (con alta densidad energética), que son cariogénicos y obesogénicos. La ingesta frecuente de grandes cantidades de carbohidratos, comunes en la dieta de niños con bajo nivel socioeconómico, confirma que el sobrepeso/obesidad se puede presentar en niños con bajo nivel socioeconómico ${ }^{(4,5,19,25)}$.

Estos hábitos dietéticos explicarían por qué la mayoría de niños con sobrepeso/obesidad presentan caries dental. Esta situación es reflejada en algunos estudios que apoyan una asociación positiva entre caries dental e IMC $(29,30)$. Un estudio sugiere que los niños con sobrepeso/obesidad tienen un riesgo de caries dental alto porque tienen un flujo salival disminuido(39), situación que también se encuentra en niños con bajo peso y que se asocia a malnutrición por deficiencia proteica ${ }^{(40)}$. Los niños obesos podrían sufrir de malnutrición por deficiencia de proteínas si consumen una dieta con alto contenido de carbohidratos y alimentos procesados ${ }^{(13)}$.

La evidencia que describe una relación inversa entre caries dental e IMC (niveles altos de caries dental asociados a bajo peso o desnutrición), viene de estudios realizados en países en vías de desarrollo y con muestras con caries de infancia temprana severa o s-ECC ${ }^{(13,15-17,20)}$. La caries de infancia temprana severa puede reducir la capacidad para alimentarse del niño, especialmente cuando la enfermedad se asocia con dolor, malestar e incomodidad. El sueño interrumpido inducido por el dolor dental puede afectar la producción de glucocorticoides resultando finalmente en una ganancia inadecuada de peso $(17,20)$. Esta situación se apoya en estudios en los que luego de la rehabilitación dental, los niños con caries de infancia temprana severa mostraron un incremento en la velocidad de crecimiento corporal al ser comparados con los controles ${ }^{(21,26-28)}$.

La desnutrición también puede predisponer a caries dental. Las deficiencias en alimentos ricos en 
proteínas pueden conducir a desnutrición proteica, disminución del flujo salival, altos niveles de caries dental y crecimiento insuficiente. Hay evidencia de que la desnutrición crónica, particularmente durante los primeros años de vida, incrementa la susceptibilidad a caries dental en la dentición primaria (por ejemplo, a través de hipoplasia del esmalte e hipofunción salival) $(40,41)$.

En la muestra estudiada no se encontró asociación entre los parámetros antropométricos y caries dental coincidiendo con los resultados de estudios previos $(23,28,38)$, situación que probablemente se deba a factores metodológicos del estudio como la falta de muestra que no permite representar adecuadamente todas las categorías de IMC ${ }^{(13,17)}$.

La alta prevalencia de caries dental (73\%) fue relativamente próxima a lo reportado en otros estudios en poblaciones similares ${ }^{(33,34,42,43)}$, reconociendo necesidades urgentes de tratamiento y la falta de acceso a servicios de salud y atención odontológica. El bajo nivel educativo, higiene oral deficiente, la ingesta de una dieta altamente cariogénica y el acceso limitado a medios de saneamiento básico son característicos de poblaciones con un entorno socioeconómico desfavorecido y tienen un claro impacto en el desarrollo de caries dental. A menudo, los niños provenientes de comunidades desfavorecidas presentan niveles de caries dental significativamente mayores $^{(28,44,45)}$. De manera similar, por una mayor capacidad adquisitiva y exposición aumentada a carbohidratos fermentables, los niños de hogares con un mayor nivel socioeconómico podrían encontrarse en un mayor riesgo de caries dental e incluso de sobrepeso/obesidad ${ }^{(5,13)}$.

En muchos países de bajos y medianos ingresos, las piezas dentarias afectadas por caries no se tratan debido al acceso limitado a servicios de salud. El índice pufa describe las consecuencias de las etapas avanzadas y no tratadas de la lesión cariosa ${ }^{(10,11,35)}$. En este estudio se encontró una prevalencia del índice pufa de $33 \%$, un porcentaje más alto que el encontrado por Figuereido et al. ${ }^{(46)}$ en niños entre 5 a 6 años en Brasil (23\%), pero menor que el descrito por Baginska et al. ${ }^{(11)}$ en niños polacos $(43,4 \%)$.

La prevalencia hallada fue relativamente próxima a la reportada por otros estudios similares en otros países en vías de desarrollo, como lo reportado por Mehta y Bahlla en India (33\%) y Thekiso $\mathrm{M}$ et al. en Sudáfrica (33\% para niños de 4 y 5 años y $41 \%$ para niños de 6 y 8 años ${ }^{(47,48)}$. La prevalencia de pufa según la edad mostró una diferencia significativa $(p=0,013)$, siendo esta mayor a los $6(45,7 \%)$ que a los 5 años (22\%), similar a lo reportado por otro estudio ${ }^{(48)}$.
Los hallazgos del presente trabajo y de estudios semejantes reflejan la poca atención a la salud oral de esta población escolar, probablemente debido a la falta de conocimiento de los padres para reconocer la enfermedad en etapas iniciales, priorización de otras necesidades y falta de acceso a servicios de atención odontológica. En esta situación, las lesiones incipientes progresan rápidamente a lesiones cavitadas e irreversibles, por falta de tratamiento oportuno para revertir este proceso. Los niños que provienen de entornos desfavorecidos presentan alta prevalencia de caries dental y acceso limitado a tratamiento odontológico $(2,6,18,19,44)$.

La mayor limitación del presente estudio es el tamaño de muestra. Estudios posteriores deben considerar el impacto de variables como higiene oral, exposición a fluoruros y dieta debido a la naturaleza etiológica multifactorial de la caries dental. El diseño metodológico de corte transversal empleado no es el más apropiado para establecer una asociación de tipo causal. Se requieren estudios longitudinales para explorar una relación de causalidad entre caries dental y crecimiento corporal en la infancia y adolescencia. Los resultados del presente estudio podrían ser tomados como referencia para estudios posteriores que permitan identificar posibles variables intervinientes en la relación entre la caries dental y los parámetros antropométricos en niños peruanos.

La muestra evaluada presentó una alta prevalencia de caries, sobretodo en el grupo clasificado como "adecuado". Los resultados del presente estudio indicaron que no existe asociación significativa entre los indicadores antropométricos y la prevalencia de caries dental y sus consecuencias clínicas en el grupo estudiado.

Agradecimientos: A la Dra. Shaela Sánchez por su apoyo para la realización de este estudio.

Contribuciones de autoría: ACL participó en el diseño del estudio, recolección de datos y redacción del artículo. EPT fue asesor, SLM participó en la recolección de datos; RMV realizel análisis estadístico. Todos los autores aprobaron el artículo.

Fuente de financiamiento: Autofinanciado.

Conflictos de interés: Los autores declaran no tener conflictos de interés en esta publicación.

\section{REFERENCIAS BIBLIOGRÁFICAS}

1. Bagramian RA, Garcia-Godoy F, Volpe AR. The global increase in dental caries. A pending public health crisis. Am J Dent 2009; 22(1): 3-8.

2 Edelstein, BL. The dental caries pandemic and disparities problem. BMC Oral Health 2006; 6(1): S2. 
3. Perú, Ministerio de salud. Prevalencia nacional de caries dental, fluororosis del esmalte y urgencias de tratamiento en escolares de 6 a 8,10,12 y 15 años, Perú 2001-2002. Oficina general de epidemiología y salud de las personas. Lima. 2005.

4. Bhurosy T, Jeewon R. Overweight and obesity epidemic in developing countries: a problem with diet, physical activity, or socioeconomic status? SCI WORLD J 2014.

5. Sahoo K, Sahoo B, Choudhury AK, Sofi NY, Kumar R, Bhadoria AS. Childhood obesity: causes and consequences. J Fam Med Primary Care 2015; 4(2): 187.

6. Mouradian WE, Wehr E, Crall JJ. Disparities in children's oral health and access to dental care. JAMA 2000; 284(20): 2625-2631.

7. Martins VJ, Toledo Florêncio TMM, Grillo LP et al. Longlasting effects of undernutrition. Int J Environ Res Public Health 2011; 8(6): 1817-1846.

8. Zafar S, Harnekar SY, Siddiqi A. Early childhood caries: etiology, clinical considerations, consequences and management. Int Dent SA 2009; 11: 24-36.

9. Ismail, Al. Determinants of health in children and the problem of early childhood caries. Pediatr Dent 2003; 25(4): 328-333.

10. Grund K, Goddon I, Schüler IM, Lehmann T \& HeinrichWeltzien R. Clinical consequences of untreated dental caries in German 5-and 8-year-olds. BMC Oral Health 2015; 15(1): 1 .

11. Bagińska J, Rodakowska E, Wilczyńska-Borawska M \& Jamiołkowski J. Index of clinical consequences of untreated dental caries (pufa) in primary dentition of children from north-east Poland. Adv Med Sci 2013; 58(2): 442-447.

12 Malek MT, Wright CM, Kay EJ. Childhood growth and dental caries. Community Dent Health 2009; 26(1): 3842.

13. Hooley M, Skouteris H, Boganin C, Satur J, Kilpatrick N. Body mass index and dental caries in children and adolescents: a systematic review of literature published 2004 to 2011. Sys Rev 2012; 1(1): 1.

14. Acs G, Lodolini G, Kaminsky S, Cisneros GJ. Effect of nursing caries on body weight in a pediatric population. Pediatr Dent 1992;14(5):302-305.

15. Sadeghi M, Roberts MW. The Relationship between BMI-for-age (BMI-\%) and dmft Index of 6-year-Old Children in Rafsanjan, Iran. Int J Dent Oral Health 2016; 2(2).

16. Khanh LN, Ivey SL, Sokal-Gutierrez K, Barkan H, Ngo $\mathrm{KM}$, Hoang HT et al. Early Childhood Caries, Mouth Pain, and Nutritional Threats in Vietnam. Am J Public Health 2015; 105(12): 2510-2517.

17. Alkarimi HA, Watt RG, Pikhart $H$, Sheiham A, Tsakos G. Dental caries and growth in school-age children. Pediatrics 2014; 133(3): 616-623.

18. Oliveira LB, Sheiham A, Bönecker M. Exploring the association of dental caries with social factors and nutritional status in Brazilian preschool children. Eur $\mathrm{J}$ Oral Sci 2008; 116(1): 37-43.

19. Cameron FL, Weaver LT, Wright CM, Welbury RR. Dietary and social characteristics of children with severe tooth decay. Scott Med J 2006; 51(3): 26-29.

20. Sheiham, A. Dental caries affects body weight, growth and quality of life in pre-school children. Br Dent J 2006; 201(10): 625-626.

21. Acs G, Shulman R, Chussid S, Ng M. The effect of dental rehabilitation on the body weight of children with early childhood caries. Pediatr Dent 1999; 109-113.

22 Mccabe M, Dávila-LaCruz M, Tomar S. Caries dental e índice de Masa corporal en niños de origen hispano. Rev Od Los Andes 2015; 10(1): 17-23.

23. Edalat A, Abbaszadeh M, Eesvandi M, Heidari A. The relationship of severe early childhood caries and body mass index in a group of 3-to 6-year-old children in Shiraz. J Dent 2014; 15(2): 68.

24. Chen W, Chen P, Chen SC, Shih WT, Hu HC. Lack of association between obesity and dental caries in threeyear-old children. Acta Paed Sin 1998;39: 109-111.

25. Granville-Garcia AF, de Menezes VA, de Lira PI, Ferreira JM, Leite-Cavalcanti A. Obesity and dental caries among preschool children in Brazil. Rev Salud Publica 2008; 10(5): 788-795.

26. Monse B, Duijster D, Sheiham A, Grijalva-Eternod CS, van Palenstein Helderman W, Hobdell MH. The effects of extraction of pulpally involved primary teeth on weight, height and BMI in underweight Filipino children a cluster randomized clinical trial. BMC Public Health 2012; 12(1): 725.

27. Gaur S, Nayak R. Underweight in low socioeconomic status preschool children with severe early childhood caries. J Indian Soc Pedod Prev Dent 2011; 29(4): 305309.

28. van Gemert-Schriks MC, van Amerongen EW, Aartman IH, Wennink JM, Jacob M, de Soet JJ. The influence of dental caries on body growth in prepubertal children. Clin Oral Investig 2011; 15(2): 141-149.

29. Willerhausen B, Blettner M, Kasaj A, Hohenfellner K. Association between body mass index and dental health in 1,290 children of elementary schools in a German city. Clin Oral Investig 2007; 11(3): 195-200.

30. Costacurta M, Di Renzo L, Bianchi A, Fabiocchi F, De Lorenzo A, Docimo R. Obesity and dental caries in paediatric patients. A cross-sectional study. Eur J Paediatr Dent 2011; 12(2): 112.

31. Marshall TA, Eichenberger-Gilmore JM, Broffitt BA, Warren JJ, Levy SM. Dental caries and childhood obesity: roles of diet and socioeconomic status. Community Dent Oral Epidemiol 2007; 35(6): 449-458. Bennadi D, Reddy, CVK. Oral health related quality of life. J Int Soc Prevent Communit Dent 2013; 3(1): 1-6.

32 Bennadi D, Reddy, CVK. Oral health related quality of life. J Int Soc Prevent Communit Dent 2013; 3(1): 1-6. 
33. Campodónico C, Pineda M, Chein S, Benavente L, Ventocilla M. El estado nutricional como riesgo para desarrollar caries en niños menores de cinco años de edad. Odontol Sanmarquina 2001; 1(7): 27-32.

34. Córdova D, Requejo A, Santa María F. Caries dental y estado nutricional en niños de 3 a 5 años de edad: Chiclayo, Perú. Kiru 2010; 7(2): 56-62.

35. Monse B, Heinrich-Weltzien R, Benzian H, Holmgren C, van Palenstein Helderman W. PUFA-An index of clinical consequences of untreated dental caries. Community Dent Oral Epidemiol 2010; 38(1): 77-82.

36. Onis MD, Onyango AW, Borghi E, Siyam A, Nishida C, Siekmann J. Development of a WHO growth reference for school-aged children and adolescents. Bulletin of the World health Organization 2007; 85(9): 660-667.

37. D'Mello G, Chia L, Hamilton SD, Thomson WM, Drummon BK. Childhood obesity and dental caries among paediatric dental clinic attenders. Int $\mathrm{J}$ Paediatr Dent 2011; 21: 217-222.

38. Sheller B, Churchill SS, Williams BJ, Davidson B. Body mass index of children with severe early childhood caries. Pediatr Dent 2009; 31(3): 216- 221.

39. Modéer T, Blomberg CC, Wondimu B, Julihn A, Marcus C. Association between obesity, flow rate of whole saliva, and dental caries in adolescents. Obesity 2010; 18(12): 2367-2373.

40. Psoter WJ, Reid BC, Katz RV. Malnutrition and dental caries: a review of the literature. Caries Res 2005; 39(6): 441-447.

41. Alvarez J, Caceda J, Woolley TW, Carley KW, Baiocchi $N$, Caravedo $L$ et al. A longitudinal study of dental caries in the primary teeth of children who suffered from infant malnutrition. J Dent Res 1993; 72(12): 1573-1576.

42. Villena R, Pachas F, Sánchez Y. Prevalencia de caries de infancia temprana en niños menores de 6 años de edad, residentes en poblados urbano marginales de Lima Norte. Rev Estomatol Herediana 2011; 21(2): 7986.

43. Chumpitaz-Durand R. Prevalencia e incidencia de caries a partir de vigilancia epidemiológica. Revista Kiru 2013; 10(2).

44. Prakash $\mathrm{P}$, Subramaniam $\mathrm{P}$, Durgesh $\mathrm{BH}$, Konde $\mathrm{S}$. Prevalence of early childhood caries and associated risk factors in preschool children of urban Bangalore, India: A cross-sectional study. Eur J Dent 2012; 6(2): 141-52.

45. Bhardwaj SV, Bhardwaj A. Early childhood caries and its correlation with maternal education level and socioeconomic status. J Orof Sci 2014; 6(1): 53.

46. Figueiredo MJ, De Amorim RG, Leal SC, Mulder J, Frencken JE. Prevalence and severity of clinical consequences of untreated dentine carious lesions in children from a deprived area of Brazil. Caries Res 2011; 45(5): 435-442.

47. Mehta A, Bhalla S. Assessing consequences of untreated carious lesions using pufa index among 5-6 years old school children in an urban Indian population. Indian $\mathrm{J}$ Dent Res 2014; 25(2): 150-153.

48. Thekiso M, Yengopal V, Rudolph MJ, Bhayat A. Caries status among children in the West Rand District of Gauteng Province, South Africa. SADJ 2012; 7(7):318320. 